\title{
Direct composite restoration of permanent anterior teeth uncomplicated crown fractures
}

\author{
Ashley Evans Nicholas*, Milly Armilia Andang*, Opik Taofik Hidayat* \\ Department Of Conservative Dentistry Faculty of Dentistry Universitas Padjadjaran
}

\begin{abstract}
An uncomplicated crown fracture is a fracture that involves only the tooth enamel or the dentin and tooth enamel without any damage or exposure to the pulp. Crown fracture of the anterior teeth usually caused by traumatic forces such as falls, accidents, violence, or sports activities. Traumatic injuries of the oral region frequently involve the anterior teeth, especially maxillary incisors due to the anatomic factors which may affect the functional and aesthetical values of the teeth. The objective of this literature study was to know more about uncomplicated crown fracture of the anterior teeth and its restoration. This research was a literature study performed by researching, highlighting various interesting facts and compiling the relevant published journals. The most common and ideal direct restoration of the anterior teeth was the composite resin restoration. The anterior teeth restoration was considered to be a complex and challenging case to solves due to the fact that besides reconstructing the tooth and regaining the function, the aesthetical aspect was also becoming the main objectives. The permanent anterior teeth uncomplicated crown fracture was the most common case of tooth fractures which was mainly caused by traumatic injuries such as falls, accidents, excessive forces, violence, and also sports activities. Dental injuries of the anterior teeth also affected the aesthetical properties and the function of the tooth. Composite resin restoration was able to performed directly on the permanent anterior teeth uncomplicated crown fracture.
\end{abstract}

Keywords: Direct restoration, permanent anterior teeth, trauma, uncomplicated crown fracture.

\section{INTRODUCTION}

Oral injuries are the fourth most common area of personal injuries among the age group of 7-30 years old. ${ }^{1}$ Traumatic dental injuries have become an important health problem not only because of the relatively high prevalence but also due to the fact of the large impact caused to an individual's daily life. ${ }^{2}$ Traumatic dental injuries constitute as one of the leading reason for odontological emergencies. ${ }^{3}$ The incidences of these injuries have increased during the past 10-20 years and a recent study have indicated that the number of the traumatic injuries incidences will exceed dental caries and periodontal diseases. Many authors have suggested that the permanent tooth fracture is a tragic experience for both child and parents. This condition was caused by the psychological discomfort, pain, loss of function, and poor aesthetics. ${ }^{4}$ 
The injured person and community with such injuries can cause expensive costs. Dentists can make a better assessment and carry out the effective treatment with the knowledge of potential prognosis of various treatment modalities. ${ }^{5}$ Crown fractures deserve a special attention due to their prevalence, variety of causative factors, and the diversity of clinical solutions proposed for the treatment, besides of the complicated and tricky restorations. ${ }^{6}$

There are many publications discussed the dental trauma of primary and permanent dentition. Andreason has conducted one of the most complex survey of this subject involving epidemiological, statistical, and diagnostic and treatment data from several countries, to evaluate the occurrence of traumatic dental injuries and their various etiological factors in relation to the age and gender of the patient, type and number of teeth involved, and the pattern of tooth fracture. ${ }^{7}$

The results of the study showed that the crown fractures were the most common injuries on the permanent anterior teeth caused by falls, accidents, violence, and sports activities. The traumatic coronal fracture was covered up to $92 \%$ of all permanent dentition traumatic injuries. The tooth that most commonly injured was maxillary central incisor $(77.0 \%)$ due to their protrusion and vulnerable position caused by the eruptive process, followed by lateral incisor $(23.0 \%)$. This result was supported by another study stated that the most commonly affected age group was 9-11 years old. Adolescents were more susceptible towards injury caused by an active lifestyle and higher risk tendency. The socio-behavioral and gender factors reported of increasing the predisposition of the anterior teeth traumatic injuries. The male group was having a higher prevalence of maxillary incisors traumatic injuries $(67.6 \%)$ than the female group (32.4\%).7 Men tended to do dangerous works and sports as well as more exposed to the road traffic accidents, whereas women had lower risk for traumatic dental injuries considering the current social condition and the low tendency of involving in intense outdoor activities. ${ }^{8}$

The general findings of etiologic factors were varied according to the age group studied. Falls were the most frequent cause of trauma in all age group, followed by sport activities $(18.6 \%)$, collisions $(11.9 \%)$, road traffic accidents
(9.2\%) and violence (6.2\%). These cases were also related to violence in both street and home, like children pranks, assaults, and physical abuse. ${ }^{8}$ The anatomic factors that reported increasing the risk of anterior teeth injuries and trauma were substantial maxillary incisor overjet and inadequate lip coverage of the anterior teeth. ${ }^{9}$

The most frequent injuries were Andreason's uncomplicated crown fracture involving tooth enamel and dentine without any exposure to the pulp (47.9\%). Anterior teeth traumatic injury mostly affected the maxillary central incisors and will involved the tooth enamel and dentine. Anterior teeth traumatic injuries may affected the aesthetic value and function of the teeth. Due to this factor, serious consideration should be made to determine the suitable restoration technique. ${ }^{8}$

Today's remarkable aesthetic achievement with prolonged durability can be obtained by using composite resin. ${ }^{10}$ All traumatic dental injuries need to be followed up over time. The followup procedures include clinical examination, radiographic assessment, and pulp sensibility testing. ${ }^{11}$

We found it to be challenging as well as highly interesting regarding the knowledge depth required, the intense skill needed, to perform an uncomplicated class IV crown fracture restoration on a patient successfully, as well as the detail required to maintain the aesthetical function. Thus, we wanted to learn more about the uncomplicated crown fractures and its various methods of restorations to be better to make an assessment and carry out more effective treatment with the knowledge of potential prognosis and various treatment modalities. This study was focusing on permanent anterior teeth uncomplicated crown fractures and its restoration.

\section{METHODS}

This research was a literature study performed by researching, highlighting various interesting facts and compiling the relevant published journals regarding the topic of interest.

\section{DISCUSSION}

The term fracture is a break or rupture of a part. In dental terms, it is classified as a 
traumatic injury to a tooth that manifests itself as a chip, crack, or break of a tooth. Crown fractures were the most frequent dental injuries in the permanent dentition. ${ }^{12} \mathrm{~A}$ dental crown is the natural portion of a tooth that covered by enamel which is visibly present in the oral cavity. A crown fracture was able to represent a danger risk due to the loss of the hard tooth tissue, and also the possibility of damage or injury towards the pulp. ${ }^{8}$ Falls or collisions characterized by high frequency of coronal fracture. The usual cause of a crown fracture is a sudden frontal impact, which theenergy inflicted upon the tooth structure exceeds the tensile strength of the dentine and tooth enamel.

The tooth fractured in a horizontal pattern following the direction of the enamel rods. If the impact came from another direction, other lines of fracture may seen. ${ }^{8}$ The dental crown fracture was found in more than $80 \%$ of fractured teeth, and when appropriate treatment was available and provided, the prognosis was also manageable.

Pulp's structure and status on a crown fracture's teeth depends on various factors such as: whether there was a concomitant (accompanying) luxation injury, the stage of current radicular development, dentine exposure level, and the time interval between injury and the treatment, and also the type and quality of dentinal coverage provided. ${ }^{8}$

A crown infraction defined as an incomplete fracture or crack of the tooth enamel, without any loss of the tooth structure. ${ }^{15}$ The cracks appeared as a line parallel with the enamel prisms and ended at the enamel-dentine junction. These lines may be either vertical or horizontal. A crown infraction may be the only visible sign of injury. Usually, complete crown infractions require no treatment. However, this trauma frequently associated with displacement injuries and also injury to the tooth's supporting apparatus that are detectable only by dental radiographs. The American Association of Endodontists classification had identified four types of cracks that were located in the crown root and also vertical root fractures that originate from the root.

An uncomplicated crown fracture generally described as a fracture limited to the dental crown (in certain cases can extend below the gum line and also affect the radicular part) with dentine exposure but without any pulp exposure. ${ }^{16}$ This type of injury was very common, approximately covering one-third of all dental injuries. ${ }^{17}$

A complicated crown fracture is a fracture that involves the tooth enamel and dentine with the pulp exposure. ${ }^{16}$ When exposure of the pulp occurred, the essential treatment was necessary to maintain the condition of the pulp. However, in primary teeth, most traumatic injuries on the anterior teeth with pulp exposure will become necrotic and infectious if left untreated for more than a month. ${ }^{18}$

An uncomplicated crown fracture was a fracture that involves the tooth enamel and dentine with the loss of the tooth structure but without any pulp exposure.16 Fractures without pulp exposure occurred more often than complicated crown fractures. ${ }^{13}$ The most fractured teeth were the maxillary incisors with the percentage as much as $80 \%$ of the central incisors and $16 \%$ of the lateral incisors. This is due to the anterior positioning and protrusion caused by the eruptive process. The anterior teeth fracture caused by trauma was the most common problem in children and teenagers due to their active lifestyle. ${ }^{7}$

Dental injuries are inconvenient for both patients and clinicians. One challenging
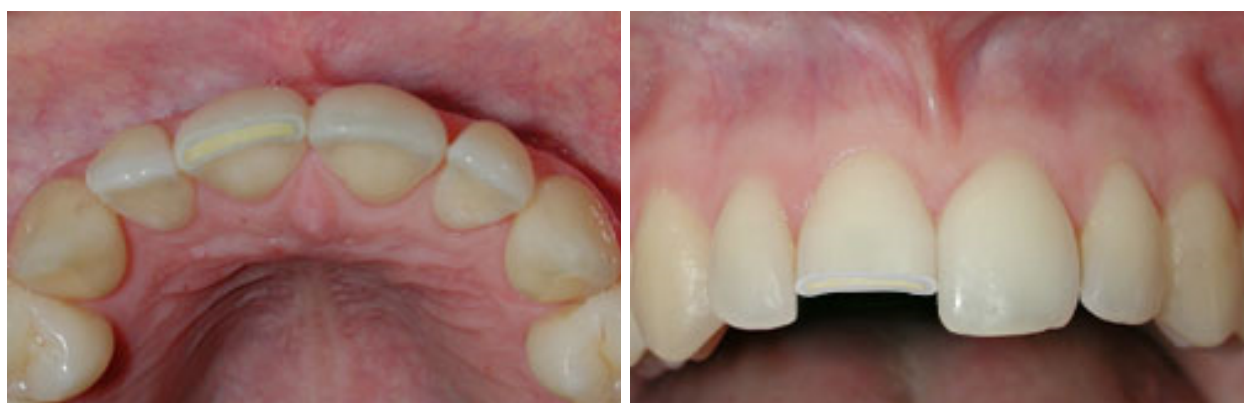

Figure 1. The permanent anterior teeth uncomplicated crown fracture without pulp exposure. ${ }^{8}$ 
situation was how to give the necessary time to conduct a qualitative evaluation of the patient's injuries. ${ }^{17}$ The first step in any diagnostic process should be gaining an accurate medical history. A comprehensive medical history and injuries chronology should obtained from the patient, parents, or any other reliable respondent. ${ }^{19}$

The goal of the treatment is to reestablishing the aesthetical aspect and also regain the form and the function of the tooth. A direct aesthetic restoration will be an effective treatment to reconstruct the permanent teeth uncomplicated crown fracture. Composite resin restoration is an ideal choice for a direct anterior restoration. Enamel fractures sometimes require only polishing or selective grinding. If the tooth fragment was not available, a restoration by class IV composite resin can performed. If the tooth fragment was available, the reattachment attempt can performed. In the condition where the fracture was close enough to the pulp, pulp protection with calcium hydroxide liner should considered. ${ }^{20}$

Composites are physical mixtures or blends of metals, ceramics, or polymers. The goal is to blend the properties of the parts to obtain intermediate properties and to take advantage of the best properties of each phase. The classic mixture for dental restorations involves ceramic particles mixed with a polymer matrix. ${ }^{20}$

The treatment of the permanent anterior teeth uncomplicated crown fracture considered as a class IV composite resin restoration. Class IV was an anterior restorative treatment, which covered a large labial, incisal and lingual surfaces and thus polymerization shrinkage will not cause any problem. ${ }^{21}$

A fracture limited to the tooth enamel may treat by only one or two method options, depends on the aesthetical concern of both of the doctor and the patient, and also the extent of the tooth loss. The first option was to recountoring the injured tooth to the adjacent tooth by polishing and selective grinding. This treatment is appropriate to eliminate the sharp enamel edges associated with the minor injuries and prevents laceration of the tongue, lips, or oral mucosa. The second option was to restore completely missing tooth structure with a Class IV composite resin. ${ }^{21}$

The maxillary incisors play a fundamental role in the facial aesthetic. Since these teeth more often suffer fractures caused by trauma, it is necessary for clinicians to know the element and basic standard for aesthetic restoration to be able to offer the patient the more suitable and pleasant smile. The size of the tooth is relevant not only for dental aesthetics but also for the overall facial esthetic. The dentists can use the relationship between the upper lip and the exposed portion of the tooth as a reference. An important factor in this proportion is that the incisal edge exposure, especially in the younger patients. During the young age, the incisal edge of upper incisor is approximately 2 to $3 \mathrm{~mm}$ longer than the upper lip line at the rest position. The visibility of the tooth when lips and mandible are at a rest position, was an important factor of dental aesthetics. Another important marker was a determination of the size of the upper central incisors that usually present the same incisal cervical length as the canines. ${ }^{21}$

The ideal form of any restoration is the patient's natural teeth arrangement. The teeth from the opposite arch (canine or incisors) could become the standard form in the cases where the tooth form has lost due to the traumatic injuries. The face morphology was also able to help in developing the tooth form. The natural tooth form divided into three categories which were square, triangular, and ovoid. ${ }^{21}$ The anterior teeth anatomy should also performed with precise detail. The distal incisal edge is more convex than the mesial incisal edge, and the surface of the lateral incisor also has the flat shape compared to the central incisors. Altering the shape of the incisal embrasure may change the width perception. All of these considerations are valuable in helping the clinician to devise a better restoration. ${ }^{21}$

The best restorative result achieved when a rubber dam applied before preparation. The restorative preparation mainly aims to establish micromechanical retention. This retention gained by doing a $45^{\circ}$ bevel created roughly $1 \mathrm{~mm}$ wide along the entire cavosurface margin. ${ }^{21}$ The bevel is located entirely inside the tooth enamel and should not extend into the dentine. The purpose of bevelling was to create a sufficient area for acid etching and to provide a suitable area for unfilled resin penetration and remove unsupported enamel. Clinically, the bevel affects the increase of the micro-retention of the restoration. ${ }^{21}$ The 
tooth preparation was performed by using the suitable burs.

Dentinal coverage was necessary to protect the pulp vitality before composite applied. ${ }^{8} \mathrm{~A}$ hard-set of calcium hydroxide liner is placed in an exposed dentine, and the tooth form was restored with a composite restoration. This traditional technique has well studied, but current evidence suggested that water-soluble calcium hydroxide has made the dentinal tubules fluid of the exposed fractured site interfered with the calcium hydroxide material, resulted in the dissolution and loss of protective function over time (Fonseca, 2006). The use of calcium hydroxide mostly recommended when the remaining dentine was within $0.5 \mathrm{~mm}$ of the dental pulp. ${ }^{13}$

The tooth enamel is etched using an acid etching to ensure the microporosity in order to increase the retention of the composite restoration. This condition can be accomplished by acid conditioning of the tooth enamel with $37 \%$ phosphoric acid for 15 seconds that produces microporosities on the surface of the residual enamel tooth structure. ${ }^{13} \mathrm{~A}$ matrix strip was then placed in the interproximal between the prepared tooth and the adjacent tooth after the enamel was etched. The most common matrix is a clear Mylar ${ }^{\circledR}$ matrix strip. ${ }^{22}$

The bonding agent was then applied to the etched enamel surface where they can penetrate into the microporosity produced by the etching technique (Hovland, 2006). The resin bonding agents are fluid, resinous materials designed to improve the bond between a viscous composite resin and the microporosities of the etched enamel. ${ }^{22}$

The composite then placed using an incremental or layering method, starting from

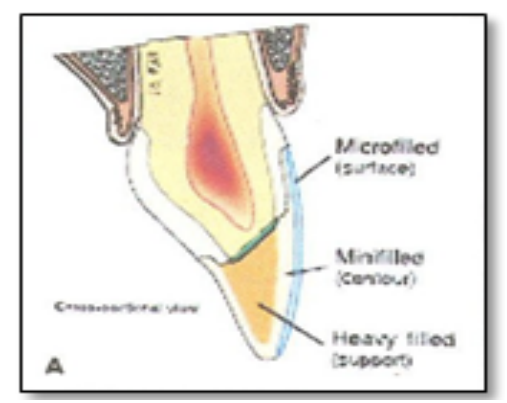

Figure 2. Placement of layers of different composite types in a large restoration. ${ }^{22}$ the hybrid composites in the internal layer and microfilled composite for the external layer to produce a shiny and smooth surface restoration. Optimal polish can be obtained with the use of the microfilled composite resin. ${ }^{21}$ The incisal edge is translucent and frequently seen in children and teenagers, so it is necessary to apply the translucent composite on the incisal edge. A larger class IV restoration can involve all three composite materials which can be heavy filled, minifilled and microfilled.

The composite was then cured in 1-2 mm increments. After placing the external layer, the Mylar ${ }^{\circledR}$ matrix strip was removed carefully before the cure, and proximal carver was used to carefully smoothed and shaped the areas with a poor access such as the gingival and the proximal margin. ${ }^{22}$ The resin was polymerized for 20 seconds before finishing and left undisturbed for at least 10 minutes to allow the resin to polymerize more completely.

Another technique published by Pereira et al., to reconstruct the anterior fractured teeth by using a reference guide from a model, which makes it possible for the clinician to increase the chance of success by planning the procedure in detail. ${ }^{23}$

The model was constructed with an alginate mould during the first visit. The fractured tooth was recognised by using progressive waxing to rebuild the size and shape of the fractured teeth. The model was then moulded again with a heavy silicone based material to obtain a reference guide. ${ }^{23}$

The restorative material and colour were selected and noted in the patient record. During the next session, the mould of the reconstructed teeth was cut into two halves, in the vestibular

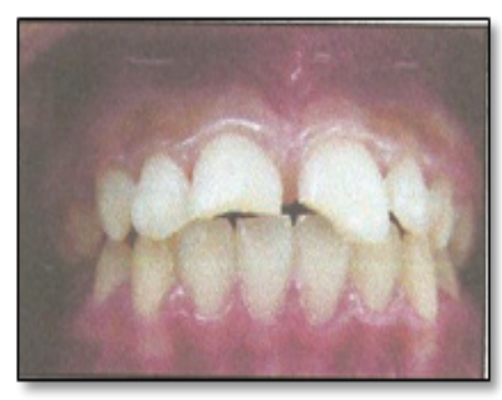

Figure 3. Uncomplicated crown fracture. ${ }^{23}$ 
and palatal part. The palatal portion of the mould would fit perfectly of the palatal aspect of the teeth to be reconstructed, thus serving as a reference guide.

The adhesive protocol was performed in accordance with the composite manufacturer's specifications. The reference guide was then fitted to the palatal surface of the teeth, and the restorative material was applied in the incremental layer to the palatal portion of the reference guide. Then the incisive material was applied to the incisal portion. Polymerization was applied for 20 seconds. Finally, the crown was reconstructed before the finishing, polishing, and adjustments.

The finishing refers to all procedure associated with contouring and eliminating excess at the margins. ${ }^{22}$ After curing lights of 20 seconds per layer, the surface was defined with a finishing diamond bur, and then the polishing was done using finishing discs. Discs play different roles in contouring and finishing of the anterior teeth. They were used for removing excess composite, shaping composite and defining anatomical structures and features. Creating a textured surface required finishing and polishing the proximal and labial surface to create an ideal contour surface texture and to place conservative development groove using a finishing discs. ${ }^{22}$

Polish relates to some terms, such as surface smoothness, shiny, or gloss. Final polishing, with the use of composite resin, can performed with abrasive slurries. ${ }^{16}$ Polishing paste such as aluminum oxide powder can be used on microfilled composites and some small particle hybrid using polishing cusps and wheels. ${ }^{22}$ Plastic polishing strip was also used for finishing and polishing. The strip provides a smooth surface for microfilled composites, and an excellent material for final finishing of proximal areas, and also tear-resistant. The microfilled composites that were placed as an external layer on the hybrid composites improve esthetic values after polishing. Finally, follow up procedures includes a clinical examination, radiographic assessment, and pulp testing should be carried out after one week, one month and then after three months if necessary.

Reattachment of the fractured segment using dentine bonding agents can be performed to restore the tooth and reduce microleakage around the restoration. There was no change in the bond strength with the use of the dentine bonding agent compared to the standard enamel bonding techniques. For deep dentine fractures, however, a calcium hydroxide hard-set liner was still the popular choice for treatment. ${ }^{16}$ Firstly, the tooth fragment was checked and confirmed whether it still fits exactly with the remaining tooth.

The placing of calcium hydroxide liner over the exposed dentine was performed if the remaining dentine was about $0.5 \mathrm{~mm}$ from the dental pulp. Preparation for the teeth was done by bevelling the remaining tooth and the tooth fragment.

A metal matrix band was used for an accurate reposition the tooth fragment to the remaining tooth. The etching and bonding procedure carried out by application of etchant and bonding agent to both original tooth and the tooth fragment as recommended by the manufacturer of the material used. The composite resin was then applied to the surface of the fracture. ${ }^{24}$

The fractured surface with composite resin reattached to the remaining tooth then followed by tightening using the matrix band. By doing this, the tooth fragment was naturally repositioned mesiodistally and buccolingually. Careful attention has to be done to the placement of the tooth fragment to ensure that it does not extrude coronally. Excessive composite resin should removed as well as any restoration defects and followed by light curing for 20 seconds from the incisal edge. After the removal of the matrix, the restoration will be reshaped and polished.

\section{CONCLUSION}

Composite resin restoration can be performed directly on the permanent anterior uncomplicated crown fracture with great success that allowed aesthetical qualities to be ensured and also restored the teeth function in just one visit with a good prognosis.

\section{REFERENCES}

1. Rajab LD. Traumatic dental injuries in children presenting for treatment at the Department of Pediatric Dentistry, Faculty of Dentistry, University of Jordan, 1997-2000. Dent 
Traumatol. 2003 Feb;19(1):6-11.

2. Traebert J, Peres MA, Blank V, Boell Rda S, Pietruza JA. Prevalence of traumatic dental injuries and associated factors among 12-yearold school children in Florianopolis, Brazil. Dent Traumatol. 2003 Feb;19(1):15-8.

3. Tapias MA, Jimenez-Garcia R, Lamas F, Gil AA. Prevalence of traumatic crown fractures to permanent incisors in a childhood population: Móstoles, Spain. Dent Traumatol. 2003 Jun;19(3):119-22.

4. Andreasen JO, Andreasen FM, Andersson L. Textbook and color atlas of traumatic injuries to the teeth. $4^{\text {th }}$ ed. Copenhagen: Blackwell Munksgaard; 2007.

5. Zuhal K, Semra OE, Huseyin K. Traumatic injuries of the permanent incisors in children in southern turkey: A retrospective study. Dent Traumatol. 2005 Feb;21(1):20-5.

6. Castro JC, Poi WR, Manfrin TM, Zina LG. Analysis of the crown fractures and crown root fractures due to dental trauma assisted by the Integrated Clinic from 1992 to 2002 . Dent Traumatol. 2005 Jun;21(3):121-6.

7. Ozel E, Cildir A, Ozel Y. Re-attachment of Anterior Tooth Fragment using a Self-etching Adhesive : A case report. J Contemp Dent Prac. 2008 Jan 1;9(1):1-7.

8. Andreasen JO, Andreasen FM, Andersson L. Textbook and color atlas of traumatic injuries to the teeth. 4th ed. Copenhagen: Blackwell Munksgaard; 2007.

9. Baldava P, Anup N. Risk factor for traumatic dental injuries in an adolescent male population in India. J Contemp Dent Prac. 2007 Sep 1;8(6):35-42.

10. Valceanu AS. Ultraconservative and Minimally Invasive Esthetic Restoration of Crown Fracture. J Cosmetic Dent. 2006 Summer;22(2):100-7.

11. Moule A, Moule CA. The endodontic management of traumatized permanent anterior teeth ; A review. Aust Dent J. 2007
Mar;52(1 Suppl):S122-37.

12. Websters, Merriam. Dictionary and thesaurus. springfield: Encyclopedia britannica publications; 2007.

13. Hovland EJ, Gutman J, Dumsha TC. Traumatic Injuries to Teeth (Dental clinics of North America) Unknown Binding. $3^{\text {rd }}$ ed. Philadelphia: W.B. Saunders Co.; 2006.

14. Roberts GJ, Longhurst P. Oral dental trauma in children \& adolescents. Oxford: Oxford University Press; 1996.

15. Blanco L, Cohen S. Treatment of crown fracture with exposed pulps. J Calif Dent Assoc. 2002 Jun;30(6):419-25.

16. Fonseca $R$, Dexter Barber $H$, Powers $M$, Frost DE. Oral maxillofacial trauma. $4^{\text {th }}$ ed. Philadelphia: Saunders-Elsevier; 2012.

17. Berman LH, Hargreaves KM, Cohen SR. Pathways of the Pulp. $10^{\text {th }}$ ed. St. Louis: Mosby-Elsevier; 2011.

18. Moule A, Moule CA. The endodontic management of traumatized permanent anterior teeth ; A review. Aust Dent J. 2007 Mar;52(1 Suppl):S122-37.

19. Hupp JR, Tucker MR, Ellis E. Contemporary oral maxillofacial surgery. $4^{\text {th }}$ ed. St. Louis: Mosby-Elsevier; 2008. p. 502-17.

20. Heymann H, Swift E Jr, Ritter A. Sturdevant's art and science of operative dentistry. $6^{\text {th }}$ ed. St. Louis: Mosby-Elsevier; 2012.

21. Geissberger M. Esthetic dentistry in Clinical Practice. Hoboken: Blackwell Publishing; 2010.

22. Albers HF. Tooth colored restoratives: Principles and Techniques. $9^{\text {th }}$ ed. London: BC Decker Inc; 2002.

23. Dos Santos PM, Maia LC. The reference guide. a step by step technique for restoration of fractured anterior permanent teeth. J Canad Dent Assoc. 2005 Nov;71(9):643-6.

24. Sukiboshi M. Treatment planning for traumatized teeth. Michigan: Quintessence Publishing Co.; 2008. 